\title{
Current neuromonitoring techniques in critical care
}

\author{
Anette Ristic ${ }^{1}$, Raoul Sutter ${ }^{1,3}$, Luzius A. Steiner $^{1,2}$
}

\begin{abstract}
Early detection of secondary events is a major target of neuromonitoring in critically ill patients. Intracranial pressure (ICP) and cerebral perfusion pressure are the most widely accepted neuromonitoring parameters. Many studies have shown both to be related to mortality after traumatic brain injury. However, the benefit of ICP monitoring has not been established by a randomized controlled trial, and the efficacy of ICP-guided management has indeed been challenged. This review considers current neuromonitoring techniques in critical care medicine.
\end{abstract}

Key words: Monitoring, neurocritical care, secondary brain injury

\section{INTRODUCTION}

Primary damage incurred in traumatic brain injury (TBI) is defined by the accident and impact itself and cannot be influenced by medical treatment. Neurocritical care must focus on treating the secondary injury resulting from swelling, metabolic derangements and ischaemia, which endanger formerly intact brain tissue. The degree of secondary injury has a critical impact on outcome, ${ }^{[1]}$ and early detection of these secondary events is a major target of neuromonitoring. The most widely accepted neuromonitoring parameters, intracranial pressure, cerebral perfusion and seizure activity, have been shown in many studies to be related to mortality after traumatic brain injury (TBI). ${ }^{[2-5]}$

This review considers current neuromonitoring techniques in critical care medicine.

\begin{tabular}{|l|l|}
\hline \multicolumn{2}{|c|}{ Access this article online } \\
\hline Quick Response Code: & Website: \\
\hline & www.jnaccjournal.org \\
\hline & \\
\hline
\end{tabular}

\section{Clinical assessment}

An altered state of consciousness is often the first sign of a clinical deterioration caused by acute brain dysfunction. A standardized scoring system can help to compare current neurological status to prior assessments, thus allowing for detection of aggravation as early as possible. The Glasgow Coma Scale (GCS) is a widely used scoring system for this purpose. It consists of three components: Assessing eye-opening, verbal and motor responses, the latter being the most important regarding outcome after TBI. The GCS is easy to use and has a high interobserver reliability. The more recently developed Full Outline of UnResponsiveness score (FOUR Score) evaluates brain stem reflexes and respiration. ${ }^{[6]}$ Thus, more subtle changes in neurological status can be detected; likewise more information can be collected in deeply comatose patients. The FOUR score also allows the explicit testing of eye movements or blinking, facilitating detection of a locked-in state. As a verbal response is not assessed, the FOUR score avoids the problem of underrating intubated but alert patients, a drawback of the GCS.

\section{Intracranial pressure monitoring}

Intracranial pressure monitoring is the most widely used technical neuromonitoring device. According to the Monro-Kellie doctrine, ${ }^{[7]}$ the incompressible skull, the blood, cerebrospinal fluid (CSF) and brain tissue exist in a state of volume equilibrium, such that any

${ }^{1}$ Surgical Intensive Care Unit, Anesthesiology, ${ }^{2}$ Department of Anesthesia, Surgical Intensive Care, Prehospital Emergency Medicine and Pain Therapy, ${ }^{3}$ Department of Neurology, Division of Clinical Neurophysiology, University Hospital Basel, Basel, Switzerland

Address for correspondence:

Prof. Luzius A. Steiner, Department of Anesthesia, Surgical Intensive Care, Prehospital Emergency Medicine and Pain Therapy, University Hospital Basel, Basel, Switzerland. E-mail: luzius.steiner@usb.ch 
increase in volume of one of the cranial constituents must be compensated by a decrease in volume of another. The initial compensatory mechanism for intracranial volume expansion following TBI or ischaemic oedema, for example, would be a reduction in CSF. Once all compensatory mechanisms have been exhausted, ICP rapidly rises resulting in decreased cerebral perfusion pressure (CPP) as demonstrated by the formula $\mathrm{CPP}=\mathrm{MAP}-\mathrm{ICP}$. MAP denotes mean arterial pressure. In the absence of autoregulation, CPP determines cerebral blood flow (CBF) and, therefore, oxygen supply.

ICP must be measured directly as it cannot otherwise be estimated by any clinical feature or computed tomography (CT). Normal ICP depends on age and body positioning, and ranges from 7 to $15 \mathrm{mmHg}$ in healthy adults. Above 20-25 mmHg, aggressive treatment is started in most intensive care units (ICU). Lower thresholds have been suggested for children. ${ }^{[8]}$

Currently, a catheter placed into one of the lateral ventricles and connected to an external pressure gauge is the most reliable and low-cost method for ICP monitoring [Figure 1]. This is the most frequently used method as it measures global ICP, provided there is no obstruction to CSF flow. It also allows therapeutic drainage of CSF to reduce ICP, and can be recalibrated in situ. However, ventricular catheters are associated with a higher infection risk compared to intraparenchymal catheters. These catheters can be difficult to place into brains with severe swelling.

Intraparenchymal catheters are easier to place, and infections are rare. This type uses a fibre-optic transducer or a piezo element on the tip of the catheter. Accuracy is usually good and although recalibration is not possible, baseline drift is low. ${ }^{[9]}$ However, it is possible that the pressure measured by the probe does not represent global ICP. The theoretical concept of an intracranial space as a single chamber with an equal distribution of pressure, however, is not always correct, as ICP in TBI patients for example may be unequally distributed throughout the cranial vault. Whether or not this is clinically important remains to be determined. Several studies have shown contradictory results in this matter. The risk of developing an ICP gradient may depend on the cause of damage. Focal lesions have been shown to cause an interhemispheric pressure shift, while in diffuse lesions no interhemispheric difference could be shown. Intraparenchymal probes should preferably be placed at the side of a mass lesion to avoid overestimation of CPP. ${ }^{[10]}$ An important disadvantage of intraparenchymal probes is the higher cost as well as the missing possibility of CSF drainage.

Other methods, such as epidural or subdural pressure probes are less accurate and, therefore, are rarely used.
Guidelines of the Brain Trauma Foundation recommend ICP monitoring in salvageable patients with GCS scores of 3-8 and an abnormal CT, defined as a scan showing haematomas, contusions, swelling, herniation or compressed basal cisterns. ICP monitoring is also recommended in patients with a GCS of 3-8 and a normal CT scan provided that at least two of the following criteria are fulfilled at admission: Age $>40$ years, unilateral or bilateral motor posturing or systolic blood pressure $<90 \mathrm{mmHg}$ (www.braintrauma.org). Some clinicians may choose to adapt these recommendations, e.g., they may monitor patients with a GCS score $>8$, if they will undergo major non-cranial surgery soon after admission.

Despite widespread use of ICP monitoring and clear guidelines, evidence on this matter is not straightforward. A meta-analysis published in 2010 found that ICP monitoring and aggressive treatment of intracranial hypertension in severe TBI is associated with improved outcomes. ${ }^{[11]}$ However, the benefit of ICP monitoring has never been proven in a randomised controlled trial. A study has even suggested that a CPP/ICP-oriented therapy will increase treatment intensity and respirator days without any improvement in outcome. ${ }^{[12]}$ However, it should be noted that the unmonitored group underwent intensive cross-sectional imaging and motor score assessment (GCS) instead of ICP monitoring.

The Benchmark Evidence from South American Trials: Treatment of Intracranial Pressure (BEST: TRIP) trial published 2012 ${ }^{[13]}$ raised further doubts about the uncritical application of ICP monitoring. This was a multi-centre, controlled trial in Bolivia and Ecuador in which 324 patients with severe TBI were randomly assigned to one of two specific protocols: Guideline-based management based on monitored ICP versus treatment based on imaging and clinical examination. The primary outcome was a composite of survival time, impaired consciousness, functional status at 3 and 6 months and neuropsychological status at 6 months. The study found that care concentrated on maintaining monitored ICP $<20 \mathrm{mmHg}$ was not superior to care based on imaging and clinical examination. However, the authors of the trial stated that they do not feel that given the ease and safety diminished ICP monitoring should follow this report, as "the study did not test the value of ICP monitoring per se" but "specifically compared two aggressive TBI treatment approaches, one of which was ICP-guided therapy." Given the current evidence, it seems that the value of ICP monitoring in severe TBI should rather be seen as a part of a multi-modality approach to targeted therapy rather than the only basis for decision-making. In a critique of the BEST: TRIP trial published 2013 in the BMJ, author Peter Hutchinson stated that he also believe(s) that a "normal" ICP should 
not be considered only in light of a particular cut-off value, because waveform analysis of the ICP is also important. ${ }^{[14]}$ Ongoing research has shown that ICP waveform analysis can provide information on the state of cerebrovascular reactivity (PRx index) and can be used to estimate optimal CPP levels for individual patients. ${ }^{[14]}$ It is important not to rely on one monitor alone, but rather to gain information from multimodal monitoring including haemodynamic measurements like CPP and ICP, as well as global measures of oxygenation and metabolism. These additional monitors including brain tissue oxygenation, jugular bulb saturation, near-infrared spectroscopy (NIRS), cerebral microdialysis and continuous electroencephalography (EEG) will be briefly summarised later in this text.

\section{Cerebral perfusion pressure}

$\mathrm{CPP}$ is considered an important treatment target for TBI patients in neurointensive care units. In order to calculate $\mathrm{CPP}(\mathrm{CPP}=\mathrm{MAP}-\mathrm{ICP})$, it is clinically important to set the reference point of the arterial pressure as well as for the ICP at the level of the foramina of Monro, or the external auditory meatus, as patients with brain injury are nursed in a $20-30^{\circ}$ "head-up position." MAP measured at the level of the heart as is frequently done, will overestimate CPP.

The Brain Trauma Foundation Guidelines for the Management of Traumatic Brain Injury recommend that CPP be maintained at $50-70 \mathrm{mmHg}$ in the brain-injured patient. Evidence demonstrates that a CPP $<50 \mathrm{mmHg}$ in TBI patients is associated with poor outcome. ${ }^{[15]}$ Target CPP $>70 \mathrm{mmHg}$ increases the risk of acute lung injury through the administration of large fluid volumes and vasopressors, often leading to poor outcome. ${ }^{[16]}$ Therefore, the Lund concept for the treatment of TBI targets a CPP $>50 \mathrm{mmHg}$ and includes a volume-targeted therapy to minimise increases in intracapillary hydrostatic pressure and intracerebral water content to avoid secondary increases in ICP. ${ }^{[17]}$ Auto-regulation is impaired by too low or too high CPP. In healthy individuals, autoregulation is effective in the CPP range 50 to $150 \mathrm{mmHg}$. However, after traumatic head injury this range is typically reduced in a highly variable manner ${ }^{[18]}$ It is very likely that rather than targeting an absolute threshold for CPP, therapy would be better guided by an individualised CPP target. Optimising CPP by monitoring cerebral vascular pressure reactivity may prevent both injurious hypotension and hypertension. Bedside monitoring of pressure autoregulatory capacity is possible by various methods, with Czosnyka's pressure reactivity index (PRx) being the most frequently used. ${ }^{[19]}$ Pressure reactivity is a key component of cerebrovascular auto-regulation. The PRx can be derived through analysis of slow waves in mean arterial blood pressure MAP and ICP. In normal autoregulation,
MAP is inversely correlated with ICP. A negative PRx value indicates intact autoregulation, and a positive value signifies a non-reactive cerebral circulation [Figure 2]. PRx correlates well with transcranial Doppler ultrasonography data with abnormal values being predictive of poor outcome after TBI. ${ }^{[20,21]}$

\section{Cerebral blood flow}

CBF can be accurately measured by cerebral CT, direct angiography, or positron emission tomography (PET), but these procedures are expensive, time consuming and put patients at risk because of the need for transport, the use of contrast agents and radioactivity.

Alternatively, CBF can be measured indirectly by transcranial Doppler, which measures the velocity of blood flow in the cerebral arteries using an ultrasound probe. The flow velocity of the blood causes a phase shift in the specific sound wave frequency emitted and recorded by the probe, wherein the wave frequency is either increased or decreased in correlation with the speed of the blood. The blood flow volume can be determined if the diameter of the vessel is known. Transcranial Doppler is primarily a technique for measuring relative blood flow changes, limiting its usefulness in neuromonitoring.

Furthermore, manipulating CBF is difficult, and there is no convincing evidence that a strategy attempting to regulate $\mathrm{CBF}$ is superior to an approach using ICP or CPP to guide therapy. There is no absolute threshold for $\mathrm{CBF}$ with regard to ischaemia, and therapeutic interventions that influence the metabolic demands of the brain (e.g., sedation or hypothermia) have an unpredictable effect on CBF-related values.

\section{Jugular bulb saturation}

Measuring $\mathrm{SJO}_{2}$ by a catheter placed in the jugular bulb, which samples of blood almost exclusively drained from the intracranial circulation, is one way of gaining information about global brain metabolism and ischaemia. Jugular oximetry can provide information about cerebral oxygen extraction and the adequacy of global CBF. This, however, is only correct if coupling between flow and metabolism is intact (i.e. changes in the metabolic rate for oxygen $\left[\mathrm{CMRO}_{2}\right]$ are linked to changes in $\mathrm{CBF}$ ).

Low CBF and ischaemia increase oxygen extraction, consequently decreasing $\mathrm{SJO}_{2}$. Normal $\mathrm{SJO}_{2}$ values range from 50 to $75 \%$. Oxygen extraction, or the arterio-jugular oxygen content difference $\left(\mathrm{AJDO}_{2}\right)$, is calculated as the difference between the arterial and jugular oxygen content in paired blood samples. Normal $\mathrm{AJDO}_{2}$ values range from 4 to $9 \mathrm{ml} / 100 \mathrm{ml}$. Inherent shortcomings of this method that have led to decreased use include: (1) Only two-thirds of the sampled blood is 
drained from the ipsilateral site; and (2) there is a large inter-individual variability in cerebral venous drainage. Therefore, $\mathrm{SJO}_{2}$ and $\mathrm{AJDO}_{2}$ can only provide information about global metabolism. Smaller lesions might not be detected. A study in head-injured patients using PET to quantify the ischaemic brain volume found that on average $170 \mathrm{cc}$ of brain tissue were ischaemic at an $\mathrm{SJO}_{2}$ of $50 \% \cdot{ }^{[22]}$ Therefore, adjusting CPP/ICP management based on $\mathrm{SJO}_{2}$ results is difficult. Too low and too high $\mathrm{SJO}_{2}$ values are associated with poor outcome. ${ }^{[22,23]}$ However, the question whether treatment directed at restoring normal $\mathrm{SJO}_{2}$ improves outcome remains unanswered.

\section{Cerebral tissue oxygenation monitoring}

Cerebral tissue oxygenation $\left(\mathrm{PbtO}_{2}\right)$ is measured by a microcatheter inserted into the white matter of the frontal lobe [Figure 3]. Oxygen diffuses through a permeable membrane surrounding the probe, entering an electrolyte solution within the probe, which creates an electrical current within the solution proportional to the $\mathrm{O}_{2}$ tension of the blood/tissue being measured. Normal baseline $\mathrm{PbtO}_{2}$ values range from 25 to $35 \mathrm{mmHg}$ (3.3 to $4.7 \mathrm{kPa}$ ). Mortality increases with time at or below a $\mathrm{PbtO}_{2}$ of $20 \mathrm{mmHg}$ or with the occurrence of any $\mathrm{PbtO}_{2}$ values $\leq 6 \mathrm{mmHg} \cdot{ }^{[24]} \mathrm{PbtO}_{2}$ is strongly influenced by $\mathrm{CPP}$ and can also be used to define an individually acceptable lower limit of CPP. ${ }^{[25]}$ However, the focal nature of $\mathrm{PbtO}_{2}$ must be emphasised, as only approximately $15 \mathrm{~mm}^{2}$ of tissue around the tip is sampled. Global assumptions can only be made in normal tissue or in a diffuse injury and not in tissues at risk. Therefore, adequate positioning of the sensor becomes crucial when using $\mathrm{PbtO}_{2}$ as an endpoint of optimised CPP.

Studies suggest beneficial effects of clinical algorithms that target normalisation of $\mathrm{PbtO}_{2}$ levels. The value of adding $\mathrm{PbtO}_{2}$ measurement to the standard ICP/ CPP-guided therapy has been assessed in two prospective observational studies. Meixenberger et al., compared a group where the ICP/CPP algorithm was combined with a $\mathrm{PbtO}_{2}$ target $>1.33 \mathrm{kPa}(10 \mathrm{mmHg})$ to a historical control group with an ICP/CPP target algorithm. ${ }^{[26]}$ Although there was no significant difference in the 6-month outcome, there was a positive trend in the $\mathrm{PbtO}_{2}$-guided group. Stiefel et al., also compared an ICP/ $\mathrm{CPP}$ target group with a $\mathrm{PbtO}_{2}$-guided group targeting a $\mathrm{PbtO}_{2}$ of $>3.3 \mathrm{kPa}(25 \mathrm{mmHg})$. the $\mathrm{PbtO}_{2}$-directed protocol produced better 6-month clinical outcomes than standard ICP/CPP-directed therapy. A review on $\mathrm{PbtO}_{2}$-based therapy combined with ICP/CPP-based therapy published 2012 suggests that this combination is associated with better outcome after severe TBI than ICP/ CPP-based therapy alone. ${ }^{[27,28]} \mathrm{PbtO}_{2}$ measurements may contribute to the prevention of secondary injury after TBI and may allow treatment adaptation tailored to the individual needs. Brain Trauma Foundation guidelines recommend monitoring $\mathrm{PbtO}_{2}$ as a complement to ICP/ $\mathrm{CPP}$-guided care in patients with severe TBI. Although no specific treatment protocol exists, the lower threshold for critical ischaemia has been defined as $<2 \mathrm{kPa}(15 \mathrm{mmHg})$. MAP and CPP are important determinants of $\mathrm{PbtO}_{2}$. However, we have to keep in mind that other factors, such as $\mathrm{pCO}_{2}, \mathrm{pO}_{2}$ and haemoglobin significantly influence $\mathrm{PbtO}_{2}$ as well.

\section{Microdialysis}

Cerebral microdialysis can be used as a bedside monitoring to detect cerebral hypoxaemia on a cellular level by measuring the energy substrate glucose and metabolites like lactate and pyruvate. A dialysis catheter $(\varnothing 0.9 \mathrm{~mm})$ is introduced into the brain parenchyma [Figure 4], and by calculating the lactate-pyruvate ratio, information on the brain's redox state, a marker of mitochondrial function, can be obtained. An increased lactate/pyruvate ratio and a decreased brain glucose level is associated with poor outcome after TBI and SAH. ${ }^{[29]}$ Wether or not microdialysis can be used to guide CCP managements remains to be determined. Although microdialysis can reveal important biochemical changes in injured brain tissue, some studies have found no relationship between elevated lactate/pyruvate levels and CPP changes. ${ }^{[30]}$

\section{Near infrared spectroscopy}

NIRS is a non-invasive monitoring technique that measures the relative concentrations of oxygenated to deoxygenated haemoglobin through the transmission and absorption of near-infrared light as it passes through tissue. The normal range of regional oxygen saturation $\left(\mathrm{rScO}_{2}\right)$ is generally stated to be 60 to $75 \%$. However, due to substantial inter- and intra-individual baseline variability it should rather be used to monitor trends. ${ }^{[31]}$ No absolute threshold exists for cerebral hypoxia and confounders, such as extracerebral or subdural haematoma can change the ratio of cerebral to extracerebral haemoglobin and, thus, offset tissue oxygen saturation values by a variable amount. ${ }^{[32]}$ Some clinical studies have shown the ability of cerebral oximetry monitoring to uncover otherwise clinically silent episodes of cerebral ischaemia in a variety of clinical settings despite the above-mentioned limitations. ${ }^{[33]}$

\section{Conservative and quantitative \\ electroencephalography}

EEG provides a continuous real time, non-invasive measure of brain function. When CBF is reduced, changes occur both in metabolic and electrical activity. The classical indications of EEG are for detecting seizures and for the prognosis of coma. A recently published review summarises the utility of the current 


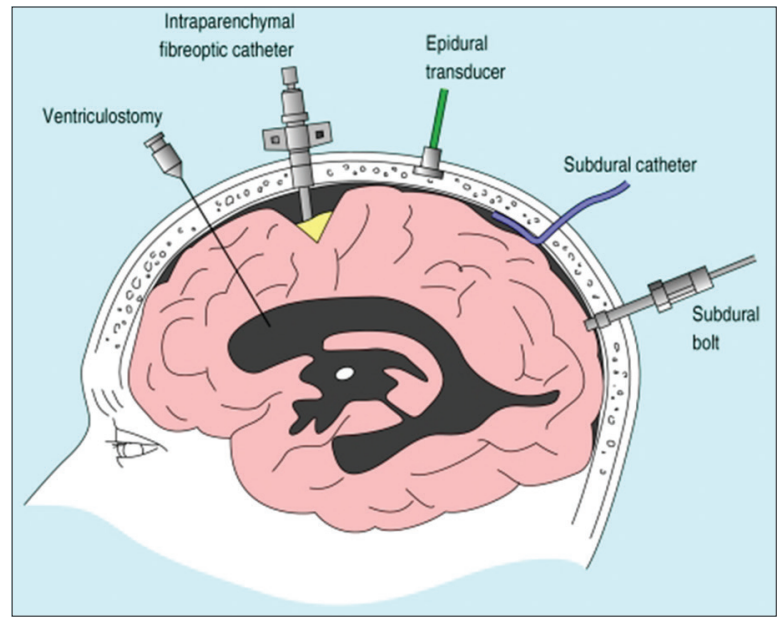

Figure 1: Intracranial pressure monitoring

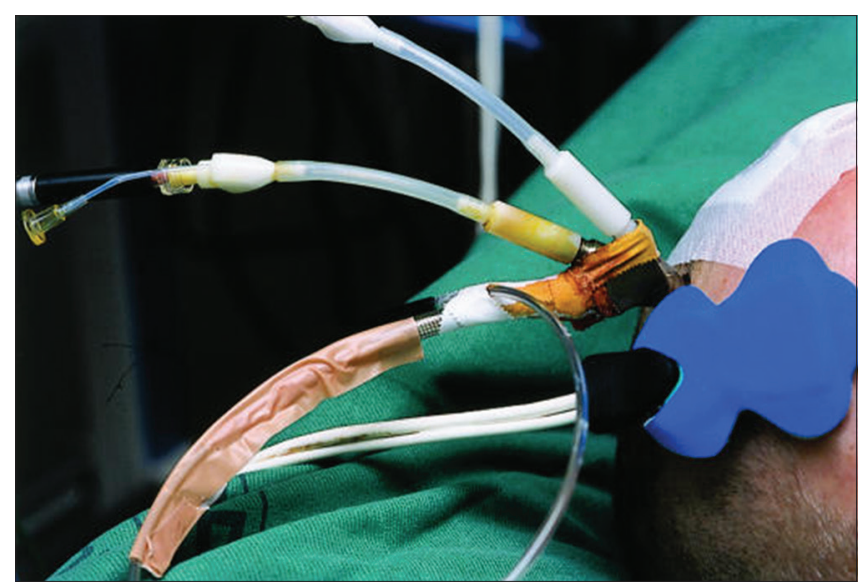

Figure 3: Brain tissue oxygenation monitoring

EEG recommendations to detect non-convulsive seizures in patients with severe brain injury in the ICU setting. ${ }^{[34]}$ However, EEG may also be useful for detection of deterioration in CBF in patients with TBI. ${ }^{[35]}$ When normal CBF declines, the EEG first loses the higher frequencies (alpha and beta bands), while the lower frequencies (delta and theta bands) gradually increase. When the CBF decreases further towards an infarction threshold, the EEG becomes isoelectric. This transition from ischaemia to infarction may provide a window of opportunity to treat. However, the EEG interpretation is difficult and highly subjective. Therefore, a variety of quantitative EEG have been developed based on the Fourier transformation principle. It is well beyond the scope of this review to explain the mathematical background, and the reader is kindly referred to specific literature on this matter. These quantitative EEG (qEEG) derived indices such as the alpha power or the alpha/delta ratio, have been used to detect delayed cerebral ischaemia in poor-grade $\mathrm{SAH}$ and severe stroke patients. ${ }^{[36]}$ qEEG is capable of detecting electrical

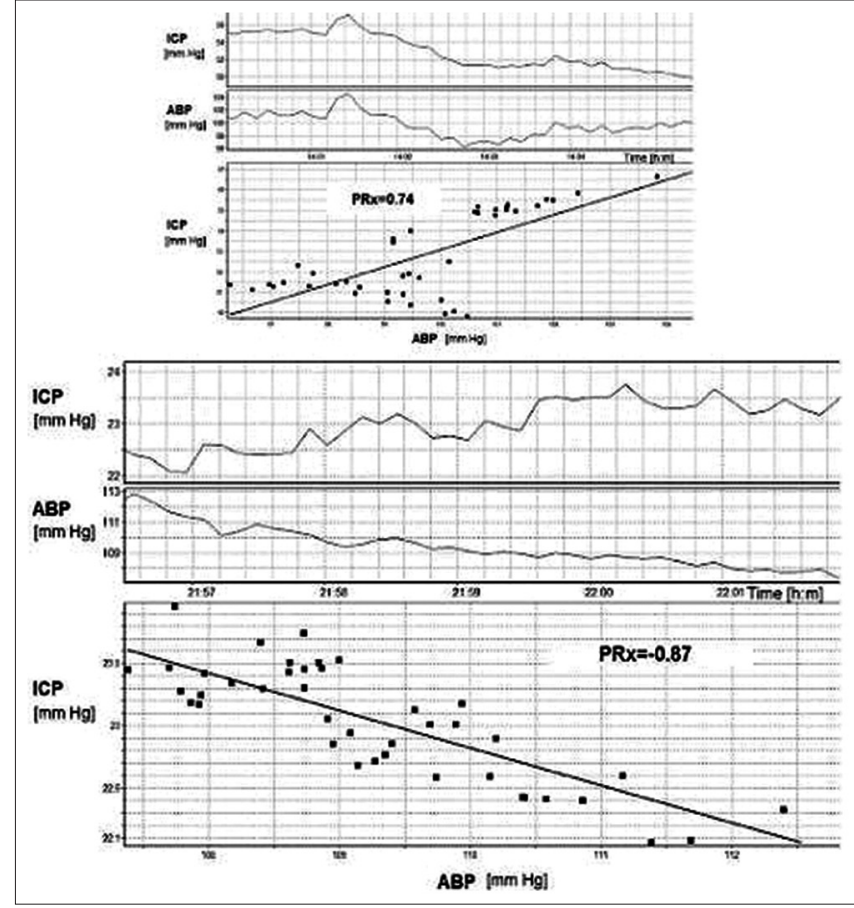

Figure 2: Intracranial and haemodynamic pressure monitoring

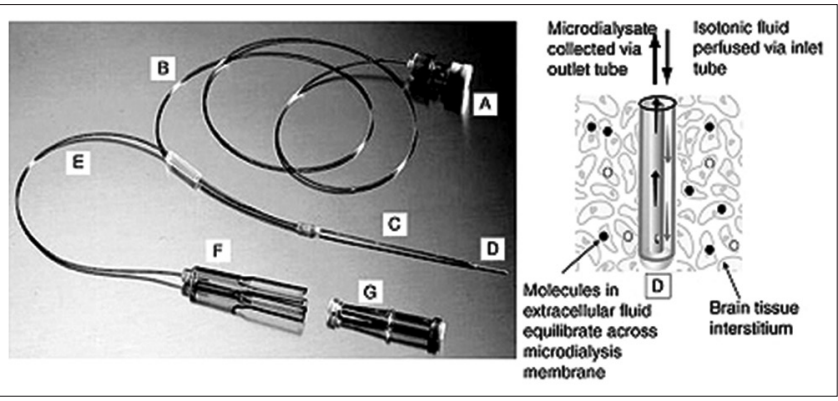

Figure 4: Brain microdialysis monitoring

changes correlating to blood flow and metabolism in as little as $28-100$ seconds. ${ }^{[37]}$ It is important, however, to consider the effect of a large number of confounders like medication (sedatives), body movements or electrical artefacts from other monitoring intensive care unit devices. In addition, increasing use of continuous EEG reveals clinically undetected epileptiform activity in up to $70 \%$ of critically ill patients depending on the underlying neurological illness ${ }^{[4,35,38]}$ and results in higher detection rates than routine EEG because of the intermittent nature of occult seizures. Using EEG monitoring, $56 \%$ of seizures are detected during the first hour of patients in a general ICU, and $88 \%$ during the first 24 hours. ${ }^{[39]}$ Furthermore, a delay in diagnosis of non-convulsive status epilepticus and prolonged seizure duration has been independently associated with increased mortality. ${ }^{[40]}$ Continuous EEG seizure detection and treatment has be associated with improved outcome. ${ }^{[40]}$ 


\section{CONCLUSIONS}

In severe brain injury, monitoring of ICP and $\mathrm{CPP}$ remains the cornerstone, particularly if a brain CT scan is abnormal despite the lack of first-class evidence due to difficulties in providing prospective randomised studies. It is important, however, not to rely on these two components alone. There is clinical evidence that demonstrates that so-called multimodal brain monitoring might help to optimise CBF and the delivery of oxygen/ energy substrate at the bedside, thereby improving the management of secondary brain injury. Looking beyond ICP and CPP, and applying a multimodal therapeutic approach for the optimization of $\mathrm{CBF}$, oxygen delivery and brain energy supply and early detection of clinically hidden epileptic seizures by EEG improve overall care of brain injured patients. Promising newer techniques for monitoring of oxygen and substrate delivery like $\mathrm{PbtO}_{2}$ monitoring, NIRS or microdialysis, as well as quantitative EEG will further help to guide therapy in intensive care medicine in the future.

\section{REFERENCES}

1. Chesnut RM, Marshall LF, Klauber MR, Blunt BA, Baldwin N, Eisenberg HM, et al. The role of secondary brain injury in determining outcome from severe head injury. J Trauma 1993;34:216-22.

2. Balestreri M, Czosnyka M, Hutchinson P, Steiner LA, Hiler M, Smielewski $\mathrm{P}$, et al. Impact of intracranial pressure and cerebral perfusion pressure on severe disability and mortality after head injury. Neurocrit Care 2006;4:8-13.

3. Marshall LF, Smith RW, Shapiro HM. The outcome with aggressive treatment in severe head injuries. Part II: Acute and chronic barbiturate administration in the management of head injury. J Neurosurg 1979;50:26-30.

4. Vespa PM, Nuwer MR, Nenov V, Ronne-Engstrom E, Hovda DA, Bergsneider $M$, et al. Increased incidence and impact of nonconvulsive and convulsive seizures after traumatic brain injury as detected by continuous electroencephalographic monitoring. J Neurosurg 1999;91:750-60.

5. Vespa PM, Miller C, McArthur D, Eliseo M, Etchepare M, Hirt D, et al. Nonconvulsive electrographic seizures after traumatic brain injury result in a delayed, prolonged increase in intracranial pressure and metabolic crisis. Crit Care Med 2007;35:2830-6.

6. Bruno MA, Ledoux D, Lambermont B, Damas F, Schnakers C, Vanhaudenhuyse A, et al. Comparison of the full outline of unresponsiveness and glasgow liege scale/glasgow coma Scale in an intensive care unit population. Neurocrit Care 2011;15:447-53.

7. Mokri B. The Monro-Kellie hypothesis: Applications in CSF volume depletion. Neurology 2001;56:1746-8.

8. Chambers IR, Jones PA, Lo TY, Forsyth RJ, Fulton B, Andrews PJ, et al. Critical thresholds of intracranial pressure and cerebral perfusion pressure related to age in paediatric head injury. J Neurol Neurosurg Psychiatry 2006;77:234-40.

9. Sahuquillo J, Poca MA, Arribas M, Garnacho A, Rubio E. Interhemispheric supratentorial intracranial pressure gradients in head-injured patients: Are they clinically important? J Neurosurg 1999;90:16-26.

10. Yano M, Ikeda Y, Kobayashi S, Otsuka T. Intracranial pressure in head-injured patients with various intracranial lesions is identical throughout the supratentorial intracranial compartment. Neurosurgery 1987;21:688-92.

11. Stein SC, Georgoff P, Meghan S, Mirza KL, El Falaky OM. Relationship of aggressive monitoring and treatment to improved outcomes in severe traumatic brain injury. J Neurosurg 2010;112:1105-12.

12. Cremer OL, van Dijk GW, van Wensen E, Brekelmans GJ, Moons KG, Leenen LP, et al. Effect of intracranial pressure monitoring and targeted intensive care on functional outcome after severe head injury. Crit Care Med 2005;33:2207-13.

13. Chesnut RM, Temkin N, Carney N, Dikmen S, Rondina C, Videtta W, et al. Global Neurotrauma Research Group. A trial of intracranial-pressure monitoring in traumatic brain injury. N Engl J Med 2012;367:2471-81.

14. Hutchinson PJ, Kolias AG, Czosnyka M, Kirkpatrick PJ, Pickard JD, Menon DK. Intracranial pressure monitoring in severe traumatic brain injury. BMJ 2013;346:f1000.

15. Howells T, Elf K, Jones PA, Ronne-Engstrom E, Piper I, Nilsson $\mathrm{P}$, et al. Pressure reactivity as a guide in the treatment of cerebral perfusion pressure in patients with brain trauma. J Neurosurg 2005;102:311-7.

16. Contant CF, Valadka AB, Gopinath SP, Hannay HJ, Robertson CS. Adult respiratory distress syndrome: A complication of induced hypertension after severe head injury. J Neurosurg 2001;95:560-8.

17. Grande PO. The Lund concept for the treatment of patients with severe traumatic brain injury. J Neurosurg Anesthesiol 2011;23:358-62.

18. Aries MJ, Czosnyka M, Budohoski KP, Kolias AG, Radolovich DK, Lavinio A, et al. Continuous monitoring of cerebrovascular reactivity using pulse waveform of intracranial pressure. Neurocrit Care 2012;17:67-76.

19. Czosnyka M, Smielewski P, Czosnyka Z, Piechnik S, Steiner LA, Schmidt E, et al. Continuous assessment of cerebral autoregulation: Clinical and laboratory experience. Acta Neurochir Suppl 2003;86:581-5.

20. Czosnyka M, Hutchinson PJ, Balestreri $M$, Hiler $M$, Smielewski P, Pickard JD. Monitoring and interpretation of intracranial pressure after head injury. Acta Neurochir Suppl 2006;96:114-8.

21. Czosnyka M. Increased intracranial pressure: What to do about it and when? Crit Care Med 2013;41:688.

22. Gupta AK, Hutchinson PJ, Al-Rawi P, Gupta S, Swart M, Kirkpatrick PJ, et al. Measuring brain tissue oxygenation compared with jugular venous oxygen saturation for monitoring cerebral oxygenation after traumatic brain injury. Anesth Analg 1999;88:549-53.

23. Alten J, Mariscalco MM. Critical appraisal of Perez et al. Jugular venous oxygen saturation or arteriovenous difference of lactate content and outcome in children with severe traumatic brain injury. Pediatr Crit Care Med 2005;6:480-2.

24. Valadka AB, Hlatky R, Furuya Y, Robertson CS. Brain tissue PO2: Correlation with cerebral blood flow. Acta Neurochir Suppl 2002;81:299-301.

25. Marin-Caballos AJ, Murillo-Cabezas F, Cayuela-Dominguez A, Dominguez-Roldan JM, Rincon-Ferrari MD, Valencia-Anguita J, et al. Cerebral perfusion pressure and risk of brain hypoxia in severe head injury: A prospective observational study. Crit Care 2005;9:R670-6.

26. Meixensberger J, Jaeger M, Vath A, Dings J, Kunze E, Roosen K. Brain tissue oxygen guided treatment supplementing ICP/ CPP therapy after traumatic brain injury. J Neurol Neurosurg Psychiatry 2003;74:760-4.

27. Nangunoori R, Maloney-Wilensky E, Stiefel M, Park S, Andrew Kofke W, Levine JM, et al. Brain tissue oxygen-based therapy and outcome after severe traumatic brain injury: A systematic literature review. Neurocrit Care 2012;17:131-8. 
28. Pascual JL, Georgoff P, Maloney-Wilensky E, Sims C, Sarani B, Stiefel MF, et al. Reduced brain tissue oxygen in traumatic brain injury: Are most commonly used interventions successful? J Trauma 2011;70:535-46.

29. Timofeev I, Carpenter KL, Nortje J, Al-Rawi PG, O'Connell MT, Czosnyka $\mathrm{M}$, et al. Cerebral extracellular chemistry and outcome following traumatic brain injury: A microdialysis study of 223 patients. Brain 2011;134:484-94.

30. Vespa PM, O'Phelan K, McArthur D, Miller C, Eliseo M, Hirt D, et al. Pericontusional brain tissue exhibits persistent elevation of lactate/pyruvate ratio independent of cerebral perfusion pressure. Crit Care Med 2007;35:1153-60.

31. Thavasothy M, Broadhead M, Elwell C, Peters M, Smith M. A comparison of cerebral oxygenation as measured by the NIRO 300 and the INVOS 5100 Near-Infrared Spectrophotometers. Anaesthesia 2002;57:999-1006.

32. Highton D, Elwell C, Smith M. Noninvasive cerebral oximetry: Is there light at the end of the tunnel? Curr Opin Anaesthesiol 2010;23:576-81.

33. Bhatia R, Hampton T, Malde S, Kandala NB, Muammar M, Deasy $\mathrm{N}$, et al. The application of near-infrared oximetry to cerebral monitoring during aneurysm embolization: A comparison with intraprocedural angiography. J Neurosurg Anesthesiol 2007;19:97-104.

34. Claassen J, Taccone FS, Horn P, Holtkamp M, Stocchetti N, Oddo M, et al. Neurointensive Care Section of the European Society of Intensive Care Medicine. Recommendations on the use of EEG monitoring in critically ill patients: Consensus statement from the neurointensive care section of the ESICM.
Intensive Care Med 2013;39:1337-51.

35. Friedman D, Claassen J, Hirsch LJ. Continuous electroencephalogram monitoring in the intensive care unit. Anesth Analg 2009;109:506-23.

36. Claassen J, Hirsch LJ, Kreiter KT, Du EY, Connolly ES, Emerson RG, et al. Quantitative continuous EEG for detecting delayed cerebral ischemia in patients with poor-grade subarachnoid hemorrhage. Clin Neurophysiol 2004;115:2699-710.

37. Foreman B, Claassen J. Quantitative EEG for the detection of brain ischemia. Crit Care 2012;16:216.

38. Sutter R, Fuhr P, Grize L, Marsch S, Rüiegg S. Continuous video-EEG monitoring increases detection rate of nonconvulsive status epilepticus in the ICU. Epilepsia 2011;52:453-7.

39. Claassen J, Mayer SA, Kowalski RG, Emerson RG, Hirsch LJ. Detection of electrographic seizures with continuous EEG monitoring in critically ill patients. Neurology 2004;62:1743-8.

40. Young GB, Jordan KG, Doig GS. An assessment of nonconvulsive seizures in the intensive care unit using continuous EEG monitoring: An investigation of variables associated with mortality. Neurology 1996;47:83-9.

How to cite this article: Ristic A, Sutter R, Steiner LA. Current neuromonitoring techniques in critical care. J Neuroanaesthesiol Crit Care 2015;2:97-103.

Source of Support: Nil, Conflict of Interest: None declared. 